\title{
La critique génétique en Argentine : précurseurs, irruption et état actuel
}

\section{Élida Lois}

\section{(2) OpenEdition}

\section{Journals}

\section{Édition électronique}

URL : http://journals.openedition.org/genesis/625

DOI : $10.4000 /$ genesis. 625

ISSN : 2268-1590

Éditeur :

Presses universitaires de Paris Sorbonne (PUPS), Société internationale de génétique artistique littéraire et scientifique (SIGALES)

\section{Édition imprimée}

Date de publication : 30 octobre 2011

Pagination : 149-156

ISBN : 978-2-84050-804-5

ISSN : 1167-5101

\section{Référence électronique}

Élida Lois, «La critique génétique en Argentine : précurseurs, irruption et état actuel », Genesis [En ligne], 33 | 2011, mis en ligne le 23 octobre 2013, consulté le 24 avril 2019. URL : http:// journals.openedition.org/genesis/625; DOI : 10.4000/genesis.625

Ce document a été généré automatiquement le 24 avril 2019.

Tous droits réservés 


\title{
La critique génétique en Argentine : précurseurs, irruption et état actuel
}

\author{
Élida Lois
}

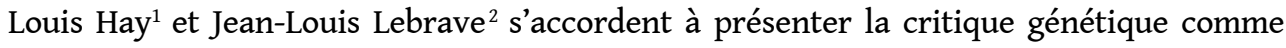
l'aboutissement d'une convergence de phénomènes culturels dont le point de départ peut être situé, pour les pays centraux, entre la fin du XVIII et le début du XIXe siècle. Différents phénomènes favorisent cette convergence: l'évolution technologique (notamment, la mise en place d'une circulation imprimée stable qui nous semble habituelle aujourd'hui mais qui à l'époque clôturait les processus séculaires de transmission textuelle manuscrite), la compilation de grandes collections de manuscrits modernes et, de manière progressive, le développement des sciences du langage et la critique littéraire. Mais en Argentine, ces composantes causales se succèdent en d'autres temps et à d'autres rythmes.

2 D'autre part, Lebrave s'arrête sur la délimitation d'un objet d'études précis : le manuscrit moderne, circonscrit au domaine privé de l'écrivain et faisant partie d'une genèse textuelle exprimée dans les témoignages successifs du processus d'écriture. Or, parce qu'il s'agit là du corpus incontournable de chaque recherche, la non-existence ou les déficits de nos institutions publiques en matière de fonds de manuscrits modernes constitue une donnée non négligeable qui renvoie aux conditions socioculturelles du pays, surtout au niveau de consolidation des politiques de la mémoire.

3 Néanmoins, avec le temps, le milieu universitaire a vu naitre des groupes de travail orientés vers ce domaine de recherches, ce qui a donné lieu, de manière progressive, à une volonté d'obtenir des manuscrits témoignant du travail d'écriture pour en faire des objets d'analyse, et même, à leur éventuelle utilisation de la part de chercheurs inscrits dans d'autres courants des études linguistiques et littéraires, ou appartenant à d'autres disciplines. Finalement, dans le cadre des Écoles d'art (notamment, des arts plastiques et de la scène), la diffusion du cadre théorique et méthodologique de la critique génétique a sollicité l'intérêt des chercheurs travaillant dans des domaines de la création régis par des processus autres que scripturaux. 


\section{La préhistoire de la génétique en Argentine}

4 On peut dire que, dans une dimension universelle, l'étude de la genèse textuelle a commencé bien avant, chaque fois qu'un lecteur critique repérait l'existence de réécritures d'un même texte en au moins deux versions (manuscrites ou éditées) réalisées par l'auteur, et qu'il y lisait des indices de sens dignes d'intérêt ${ }^{3}$. Ainsi, nous pouvons dire que le juriste, homme d'État et écrivain Juan Bautista Alberdi est devenu un précurseur inattendu des études génétiques dans l'Amérique hispanophone, lorsqu'en 1853, au beau milieu d'une célèbre polémique avec Domingo Faustino Sarmiento ${ }^{4}$, il analyse la genèse textuelle d'une des œuvres de son adversaire : Facundo o Civilización y Barbarie, un texte qui inaugure toute une tradition d'essais portant sur l'identité nationale.

Alberdi remarque que la première édition de Facundo ne devait pas être lue uniquement comme l'histoire de la barbarie et une critique des excès des caudillos argentins, mais également comme l'histoire des erreurs de la civilisation, représentée par l'élitisme du parti unitaire. Pourtant, dans la deuxième édition, suivant le conseil du leader unitaire qui s'apprêtait à reprendre le pouvoir, Sarmiento décide de supprimer une introduction dans laquelle il admettait que le pouvoir des caudillos restait l'expression habituelle de la société argentine, ainsi que les deux derniers chapitres, dans lesquels il démontrait que le libéralisme dépourvu de sens pratique était voué à l'échec. En mettant en évidence cette métamorphose politique, Alberdi (qui, de par sa philosophie politique, cherchait systématiquement à surmonter des positions antithétiques) saisit l'orientation d'un processus de genèse textuelle, et pourrait donc être considéré comme un généticien avant la lettre.

6 Si l'on en revient à l'histoire, force est de remarquer que la circulation manuscrite n'a pas cessé en Argentine au cours du $\mathrm{XIX}^{\mathrm{e}}$ siècle. Le célèbre poète gauchesco Estanislao del Campo, par exemple, faisait circuler parmi ses amis de nombreuses versions autographes de son Fausto vernaculaire. Mais ce qui semble davantage étonnant c'est l'existence constatée de versions manuscrites du Martín Fierro qui circulaient dans les provinces du nord-ouest. Rappelons qu'il s'agit là du phénomène éditorial le plus important de la littérature argentine du XIX ${ }^{\mathrm{e}}$ siècle : José Hernández a vu se suivre de son vivant douze éditions de El gaucho Martín Fierro pour un total de cinquante-huit mille exemplaires - des chiffres inouïs à l'époque -, auxquels il faut ajouter les trois réimpressions de La vuelta de Martín Fierro, plus les éditions pirates des deux œuvres qui s'imprimaient à Buenos Aires, à Montevideo et à Rosario. Mais au-delà de cette fracture centre/périphérie qui se vérifiait au sein même du pays, la professionnalisation de l'écrivain - ce phénomène social de la modernité qui est à l'origine de la valorisation du travail d'écriture, un trait avancé par Lebrave - ne devient évidente en Amérique latine que dans la première moitié $\mathrm{du} \mathrm{xx}^{\mathrm{e}}$ siècle.

7 Lebrave signale également de quelle manière l'existence de grandes collections de documents d'écriture a poussé quelques critiques à décrire du matériel de genèse. Il mentionne parmi d'autres Antoine Albalat, un expert en didactique de l'écriture et du travail du style qui, en 1903, a entamé une analyse assez systématique des manuscrits de Chateaubriand, de Hugo, de Balzac et de Flaubert.

8 Albalat a eu un disciple argentin à distance : Carlos Alberto Leumann, qui, après avoir lu ses travaux, a entrepris une analyse des brouillons de La vuelta de Martín Fierro, ce qui l'a 
conduit à décrire la matérialité de l'écriture. Sa méthodologie, pourtant, peut être réduite à une seule règle: repérer de manière exhaustive toutes les reformulations de José Hernández afin de montrer l'existence d'un chemin qui conduit à la perfection, ce qui lui permet de s'adonner ensuite à l'exaltation du résultat final ${ }^{5}$.

Leumann a projeté, en outre, dans l'étude de ces avant-textes les procédés de description linéaire typiques de la linguistique historico-comparative, qui sont à la base de la méthodologie lachmannienne; il a fait cela avec la même obstination que celle qui l'a poussé à construire un archétype textuel pour une édition critique de Martín Fierro qui scandalise aujourd'hui les sociolinguistes.

En 1943, deux ans avant cette publication qui fait état d'un critique prisonnier dans les serres du XIX ${ }^{e}$ siècle, le philologue espagnol Amado Alonso publie un travail qui a pour titre « Le manuscrit du Fausto dans la Collection Martiniano Leguizamón ». Pour ce faire, Alonso a dû se contenter d'un échantillon partiel de la genèse du Fausto: des copies autographes avec des amendements ${ }^{6}$, mais son analyse cherche à prouver que la plupart des réécritures visaient à adapter le texte non pas à la seule grammaire du monde paysan mais aussi au style fluide et naturel de la poésie populaire, plus proche de la langue courante des villes. De cette manière, dans la mesure où il met en évidence les tiraillements et l'aller-retour entre ces deux espaces culturels, cet article ébauche des idées qui seront reprises et exploitées dans la deuxième moitié $\mathrm{du} \mathrm{xx}^{\mathrm{e}}$ siècle, lorsque cette littérature sera lue comme l'expression de l'alliance des classes que sous-tend la formation de la nation.

11 Amado Alonso s'était formé à l'école philologique de Ramón Menéndez Pidal, qui avait réussi à combiner la rigueur descriptive des néogrammairiens avec la prise en compte du langage comme phénomène inséparable des processus sociaux et culturels; à cette formation de base il a su incorporer de manière originale aussi bien des conceptions idéalistes que structuralistes. Cette formation lui a permis de saisir facilement le sens d'un phénomène linguistico-littéraire de nature dynamique. Son analyse fait état de la distance qui sépare le chercheur qui manipule du matériel de genèse de celui qui l'interprète. Alonso peut donc être considéré à juste titre comme un précurseur de la critique génétique dans le domaine universitaire.

\section{L'irruption de la critique génétique et le programme international « Archivos »}

12 Il n'est pas étonnant, dans ce sens, que l'on doive à Ana María Barrenechea, l'une des principales disciples argentines d'Amado Alonso, l'introduction des études génétiques en Argentine.

13 Un déplacement entre deux perspectives opposées mais nécessaires à l'interprétation de phénomènes linguistico-littéraires, comme celle que l'on observe dans la trajectoire d'Amado Alonso, mais allant cette fois de l'objectivisme abstrait des systématisations structuralistes jusqu'à des modèles plus larges et plus souples, avait déjà entrainé un groupe de chercheurs français du CNRS qui analysaient les manuscrits de Heine, dans les années soixante, à parcourir le chemin qui conduit de la philologie traditionnelle à la critique génétique. En prenant de l'avance sur les élaborations théoriques qui allaient s'ensuivre et alors que n'avaient circulé parmi nous que le travail de Bellemin-Noël introduisant le concept d'avant-texte ${ }^{7}$ et deux recueils d'articles de membres des équipes 
du CNRS ${ }^{8}$, Ana María Barrenechea allait, pour sa part, publier dès 1983 Cuaderno de bitácora de Rayuela de Julio Cortázar'. À l'époque, prévalait encore la notion d'«avanttexte » comprise comme un complément du «texte ", comme une manière de parvenir jusqu'à lui. Dans ce contexte critique, l'analyse d'un embryon textuel de Rayuela apparaît comme l'une des premières expériences d'édition génétique et un guide pour la lecture d'une genèse textuelle.

En arpentant les sentiers fluctuants de la production littéraire à partir des papiers témoignant du travail d'écriture, Barrenechea atteint inévitablement la notion de « texte » comme " éventualité ». En d'autres termes, elle remet en question le concept de texte utilisé jusqu'à ce moment-là et elle réalise par la même occasion des apports méthodologiques. En analysant le dossier génétique réuni, elle remarque qu'il ne s'agit pas de brouillons stricto sensu mais d'un ensemble hétérogène de matériaux de genèse prérédactionnels (des esquisses de nombreux passages, des plans de certains chapitres, des listes et des portraits de personnages, des commentaires et des annotations de travaux préalables à l'écriture), qui contiennent également quelques mises en texte et quelques notes métascripturaires. Toutefois, Barrenechea ne se contente pas d'analyser une poétique de l'écriture de Cortázar à partir de sa dynamique créative : elle y perçoit le sens d'un nouveau courant dans les études philologiques et elle en théorise l'apparition. Elle le place dans le cadre général d'un mouvement scientifique qui modifie les paradigmes : la psychologie et ses progrès dans la réflexion sur les processus cognitifs ; la psychanalyse avec ses notions de pulsion, de déplacement, de condensation et ses modèles qui remettent en question l'unité du Moi; la linguistique dans son versant générativiste et son utilisation des opérations de transformation ; la sociolinguistique ; les théories du discours qui introduisent la pragmatique et envisagent l'interaction verbale comme un ensemble de stratégies en procès; les théories marxistes sur la production utilisées de manière plus ou moins réussie dans le domaine de la littérature; le déconstructionnisme de Derrida et une science littéraire qui stimule une critique de l'écriture et de la lecture. Dans ce contexte de la seconde moitié du $\mathrm{xx}^{\mathrm{e}}$ siècle, une conception rénovée de la genèse scripturaire donne lieu à l'apparition d'une authentique "critique génétique», susceptible de développer une conscience théorique et méthodologique qui réaménage les pratiques philologiques de l'époque ${ }^{10}$.

Une autre donnée importante pour mieux décrire la pratique génétique en Amérique latine est l'apparition de la collection "Archives ", éditée par l'association Archives de la littérature latino-américaine, des Caraïbes et africaine $d u x^{e}$ siècle, à partir d'un programme réalisé sous l'égide de l'Unesco.

Dans le cadre du programme international «Sauvegarde de la mémoire écrite latinoaméricaine ", l'association Archivos - dont le siège se trouvait au départ à l'université Paris X et ensuite à la Maison des Sciences de l'Homme et de la Société de l'université de Poitiers - édite la collection "Archivos ", créée et dirigée par Amos Segala jusqu'en 2005. La collection était née comme la manifestation éditoriale d'un projet international et intercontinental multidisciplinaire soutenu par l'Espagne, la France, l'Italie, le Portugal en ce qui concerne l'Europe -, par l'Argentine, le Brésil, le Costa Rica, le Guatemala, le Mexique, le Pérou - dans le cas de l'Amérique. Grâce à ce soutien, des accords organiques avec des universités de chacun de ces pays ont été signés, ce qui a permis d'entamer un projet culturel dont l'expression matérielle est représentée par l'édition de plus de soixante volumes déjà publiés. 

le séminaire sur «La méthodologie et la pratique de l'édition critique de textes modernes ", séminaire au cours duquel sont fixées les grandes lignes pour le traitement des textes, des avant-textes et des autres matériaux qui seraient édités dans le cadre du projet $^{11}$, il est vrai que l'on songe à une collection vouée à l'édition critique de textes « canoniques ", dans laquelle le matériel avant-textuel se présente comme un chemin qui conduit vers eux. Mais dans les cas où ce matériel est particulièrement intéressant il va sans dire que l'on présente également une possibilité de faire lire des genèses. Et c'est dans ce sens que le programme Archives s'est constitué comme un espace de construction d'une critique génétique latino-américaine. l'objet d'un numéro spécial de la revue Filología ${ }^{12}$, organe de l'Institut de philologie de l'université de Buenos Aires, dirigé à l'époque par Ana María Barrenechea. À l'initiative d'Amos Segala trois remarquables théoriciens de l'ITEM : Louis Hay ${ }^{13}$, Almuth Grésillon ${ }^{14}$ et Jean-Louis Lebrave ${ }^{15}$ avaient été invités à présenter des articles de fond sur le sujet, qui furent traduits en espagnol. Par ailleurs, on publia des articles de chercheurs participant au programme Archives, en même temps que d'autres dossiers génétiques d'auteurs argentins étaient l'objet d'analyse à l'occasion de ce volume. Même si l'étude de matériaux de genèse de l'écriture prévaut, un nouveau versant se dessine avec ces articles. L'experte en narration orale María Inés Palleiro explore les approches de la méthodologie génétique susceptibles d'être appliquées à d'autres types de corpus: les processus créatifs produits et enregistrés à l'oralité.

Dans ce contexte, lorsque la famille de Manuel Puig offre à l'Université nationale de La Plata les archives de l'écrivain, une équipe de chercheurs dirigés par José Amícola - qui avaient réalisé de nombreux travaux sur Puig mais à partir d'autres cadres théoriques et méthodologiques - ont tout de suite saisi la valeur et l'importance de ce corpus. À partir du chemin frayé par le programme Archives et les activités de l'Institut de philologie de l'université de Buenos Aires, ils ont entrepris une recherche inédite dans le pays: l'analyse d'un fonds important de papiers de travail d'un écrivain ${ }^{16}$.

De même, peu de temps après, est paru en Argentine le premier manuel de critique génétique écrit en espagno ${ }^{17}$, qui - tout en demeurant le seul livre consacré entièrement à ce courant critique en langue espagnole - s'est trouvé complété par un autre manuel publié par le Centre de recherches latino-américaines de l'université de Poitiers - axé entièrement autour du programme Archives ${ }^{18}$.

De 1988 à 2002, la collection " Archivos ${ }^{19}$ » a publié huit volumes comprenant des éditions critiques et génétiques et des analyses de processus d'écriture d'auteurs argentins couvrant un éventail qui commence par la littérature gauchesca - Martín Fierro de José Hernández (Élida Lois, vol. LI) -, se poursuit dans les développements postérieurs du genre - Don Segundo Sombra de Ricardo Güiraldes (Élida Lois, vol. II) -, s'arrête sur les avant-gardes du début du $\mathrm{xx}^{\mathrm{e}}$ siècle - Museo de la novela de la Eterna de Macedonio Fernández (Ana María Camblong, vol. XXV), Adán Buenosayres de Leopoldo Marechal (Jorge Lafforgue y Patricia Vila, vol. XXXI) et l'Obra completa de Oliverio Girondo (Raúl Antelo, vol. XXXVIII) -, consigne le boom de la littérature latino-américaine avec Rayuela de Julio Cortázar (Julio Ortega et Flor María Rodríguez Arenas, vol. XVI) et se rapproche du présent avec Sudeste - Ligados d'Haroldo Conti (Eduardo Romano et Miriam Goldstein, vol. XXXIV), et finalement, El beso de la mujer araña de Manuel Puig (José Amícola, Julia Romero et Graciela Goldchluk, vol. XLII).

Genesis, 33 | 2011 
Mais lorsqu'il fallut enregistrer et présenter les processus d'écriture, les responsables de la collection se sont heurtés à un problème technique : le moule simple et fonctionnel que constituait l'appareil de lecture conçu par Giuseppe Tavani s'avérait insuffisant pour rendre « lisible » un dossier génétique bien fourni ${ }^{20}$. Dans ce sens, le matériel génétique, volumineux et éblouissant, de El beso de la mujer araña a dû être accompagné d'un CD-Rom, et cette nécessité de compléter l'édition papier avec la reproduction numérique de documents et de transcriptions - ce qui entraîne de nouvelles possibilités de stockage mais aussi de recherche, de manipulation et de contraste impossibles avec les éditions traditionnelles - a inauguré une nouvelle étape dans l'histoire de la collection car dès lors que l'on conçoit l'édition génétique comme une machine servant à lire les documents archéologiques d'une production littéraire, le support électronique s'avère être le canal idéal pour atteindre cet objectif.

Après une interruption de cinq ans en raison de difficultés financières, est parue en 2008 l'édition critique et génétique de Sobre héroes y tumbas d'Ernesto Sábato (Norma Carricaburo, vol. LX), comprenant des documents intéressants sur le processus d'écriture et les reformulations enregistrées dans les versions éditées successivement. C'était la première fois que la collection se consacrait à un écrivain "vivant ", circonstance ayant ouvert la porte à des dialogues enrichissants avec l'auteur de l'œuvre étudiée. Cette possibilité a été décisive lors de la préparation du dernier volume consacré par la collection à la littérature argentine: Glosa/El entenado de Juan José Saer (Julio Premat, vol. LXI).

24 La nouvelle série de la collection a inauguré ainsi une autre étape dans ce processus d'« archivage » de la mémoire latino-américaine, grâce à la coordination scientifique de l'ITEM (CNRS) et la responsabilité éditoriale de Fernando Colla et Sylvie Josserand.

À l'heure actuelle, au sein de l'Université nationale de La Plata, Graciela Goldchluk - l'une des principales collaboratrices du projet de José Amícola - poursuit son travail dans le domaine de la génétique textuelle : elle dirige la mise en ligne des archives numériques de Manuel Puig ${ }^{21}$, tout en formant de nouveaux disciples et en les encourageant à recueillir de nouvelles archives d'écriture ${ }^{22}$. À l'Institut de philologie de l'université de Buenos Aires, María Inés Palleiro continue son travail sur les processus créatifs propres à l'oralité et elle forme des chercheurs à cette approche ${ }^{23}$. Le Centre d'études avancés de l'Université nationale de Córdoba encourage, lui aussi, le rassemblement et l'analyse d'archives écrites.

De même, depuis 2002, j'ai été chargée de l'édition des plus importantes archives de manuscrits modernes du XIX ${ }^{e}$ siècle que possède l'Argentine. Le projet d'« Édition des archives documentaires de Juan Bautista Alberdi " s'inscrit dans le programme que je dirige au sein du Conseil national de recherches scientifiques et techniques (Conicet) : "Archives de la mémoire de l'écriture argentine »; un programme que je mène à l'aide d'une équipe de chercheurs et de techniciens.

On trouve dans le fonds Alberdi ${ }^{24}$ plus d'une centaine de carnets de notes et quelques dizaines de dossiers comprenant des brouillons autographes qui contiennent des pages inédites, sa correspondance, des écrits de tierces personnes et des coupures de journaux qu'il conservait soigneusement. Compte tenu de l'importance de la figure d'Alberdi en tant qu'homme d'État, jurisconsulte et écrivain, compte tenu aussi de la répercussion de son œuvre écrite dans la formation de l'État argentin, on estime qu'il est indispensable d'enrichir la connaissance de son profil intellectuel par une analyse des tensions 
conceptuelles et idéologiques que ses réécritures mettent en évidence (et qui n'ont à ce jour jamais été étudiées), ainsi que par la reconstruction de ses échanges épistolaires (pour la plupart inédits). La Série Alberdi, constituée d'éditions critiques et génétiques de ses brouillons ${ }^{25}$ et des éditions critiques de sa correspondance inédite, est aujourd'hui éditée sous l'égide de l'Université nationale de San Martín.

Mais des archives aussi considérables permettent d'élargir l'horizon des recherches génétiques; c'est le cas d'une partie de ce fonds constituée par près de huit cents pièces épistolaires envoyées à Alberdi entre 1835 et 1884 par des femmes, offrant un large échantillon de nationalités et de secteurs sociaux, ce qui a permis à Magdalena Arnoux d'étudier la manière dont les échanges avec cette puissante personnalité masculine provoquent différentes transformations au niveau de l'écriture dans le discours épistolaire de ses correspondantes les plus assidues ${ }^{26}$.

\section{Nouvelles pratiques et autres voies d'exploration}

Encore une fois, les projets de groupes de chercheurs argentins rejoignent les objectifs du programme Archives et essaient, par la voie associative, de créer un mouvement d'intégration culturelle supranationale et la mise en place de "politiques de la mémoire ».

Les éditions critiques et génétiques sur support papier de la Série Alberdi auront pour complément la reproduction sur support numérique des manuscrits autographes de Juan Bautista Alberdi. Dans le cadre d'un accord de coopération entre l'Unsam et le Centre de recherches latino-américaines de Poitiers, il a ainsi été décidé de publier ces manuscrits dans le réseau du fonds des écrivains latino-américains que diffuse ce Centre: les "Archives virtuelles ${ }^{27}$ ", et ce matériel sera bientôt disponible pour les chercheurs qui souhaitent le consulter.

De fait, les dossiers les plus importants (comprenant un matériel prérédactionnel et rédactionnel abondant et n'excluant aucune des catégories de classement connues, multipliant les étapes dans chacune de ces catégories) n'admettent que des éditions numériques. On y inclura prochainement l'édition génétique d'un des dossiers documentaires conservés les mieux fournis : les papiers du travail d'écriture destinés à $D e$ la anarquía y sus dos causas principales, dont le texte final est un essai admirable par sa concentration discursive et son argumentation impeccable ${ }^{28}$.

Même si ce programme d'"Archives virtuelles", ouvert à la communauté latinoaméricaine, a pour point de départ un fonds d'écrivains dont ce Centre détient la propriétée ${ }^{29}$, un appel a été lancé visant à y ajouter des dépôts de documents dispersés. Parallèlement à la volonté d'aller au-delà des "archives culturelles officielles ", on ne rejette pas les admissions plurielles, tant dans le domaine de la production de sens que dans celui du canon critique qui interagit avec elle. C'est par ce biais qu'une « politique de la mémoire » est définie. Et c'est aussi pour cette raison que l'on parle d'«archives » au pluriel, car on accepte d'emblée la pluralité et la diversité et on met en relief la condition d'archives «virtuelles» en utilisant les ressources informatiques non seulement pour augmenter la capacité de stockage et les possibilités de traitement mais aussi pour éviter une muséification qui à la fois conserve et supprime.

Archiver et interpréter sont, en fait, des activités complémentaires : choisir, classer, ouvrir la possibilité de mettre en rapport, revient à fournir des propositions de lecture, et cela 
constitue déjà un premier essai d'interprétation. Mais il s'agit surtout de propositions de lecture où l'on permet aux matériaux de parler par eux-mêmes ; c'est ce que l'on comprend par l'expression production de présence, qui reconnaît l'influence de la pensée de Jean-Luc $\mathrm{Nancy}^{30}$. Dans cette ligne, l'existence d'archives n'est pas uniquement un rappel de l'impossibilité de supposer que tout ce qui a été dit a été documenté, que tout ce qui a été documenté a ensuite été archivé et que tout ce qui a été archivé a été publié ; l'existence d'archives constitue la mise en jeu permanente d'une dialectique entre les effets de " présence » et les effets de «sens».

Nous évoquions au départ le rapport intime entre les archives documentaires et les recherches génétiques, en nous limitant à la description des études portant sur les dynamiques d'écriture. Il reste néanmoins indubitable que les approches les plus récentes des processus créatifs élargissent le champ d'action des études génétiques en Argentine, tout en enrichissant le concept traditionnel d' " archives ». Et je souhaite conclure sur ce point, en reprenant une déclaration de Daniel Ferrer vieille de dix ans mais qui n'a rien perdu de sa vigueur: "La critique génétique du $\mathrm{xxI}^{\mathrm{e}}$ siècle sera transdisciplinaire, transartistique et transsémiotique ou n'existera pas $^{31}$. »

\section{NOTES}

1. Louis Hay, « Nouvelles notes de critique génétique : la troisième dimension de la littérature », Texte, nำ 5-6, Toronto, 1986, p. 313-328.

2. Jean-Louis Lebrave, «La critique génétique : une discipline nouvelle ou un avatar moderne de la philologie ? ", Genesis, nº 1, 1992, p. 33-72.

3. En 1501, par exemple, Pietro Bembo commentait déjà les nombreuses variantes du texte du Canzoniere de Petrarca.

4. Voir Alba Omil (éd.), Polémica Alberdi-Sarmiento, Tucumán, Universidad Nacional de Tucumán, 2003.

5. Carlos Alberto Leumann, El poeta creador, Buenos Aires, Sudamericana, 1945.

6. Élida Lois, "Amado Alonso, precursor de la crítica genética », Cauce, n 18-19, Universidad de Sevilla, 1995-1996, p. 401-408.

7. Jean Bellemin-Noël, Le Texte et l'avant-texte. Les brouillons d'un poème de Milosz, Paris, Larousse, 1972.

8. Louis Hay (éd.), Essais de critique génétique, Paris, Flammarion, 1979 ; Raymonde Debray Genette (éd.), Flaubert à l'œuvre, Paris, Flammarion, 1980.

9. Ana María Barrenechea, Cuaderno de bitácora de Rayuela de Julio Cortázar, Buenos Aires, Sudamericana, 1983.

10. Ibid., p. 13-19.

11. Giuseppe Tavani, "Teoría y metodología de la edición crítica ", dans Littérature latinoaméricaine et des Caraibes du XXe siècle. Théorie et pratique de l'édition critique, Amos Segala (éd.), Roma, Bulzoni Editore, 1988, p. 21-84.

12. Filología, vol. XXVII, no 1-2, Élida Lois (éd.), Buenos Aires, 1994.

13. Louis Hay, «La escritura viva », ibid., p. 5-23.

14. Almuth Grésillon, «Qué es la crítica genética », ibid., p. 25-52. 
15. Jean-Louis Lebrave, «La crítica genética : ¿una nueva disciplina o un avatar moderno de la filología? », ibid., p. 53-73.

16. José Amícola, Graciela Goldchluk, Roxana Páez y Julia Romero (éd.), Materiales iniciales para La traición de Rita Hayworth de Manuel Puig, La Plata, Centro de Estudios de Teoría y Crítica Literaria, UNLP, 1996.

17. Élida Lois, Génesis de escritura y estudios culturales. Introducción a la crítica genética, Buenos Aires, Edicial, 2001.

18. Fernando Colla (éd.), Archivos: cómo editar la literatura latinoamericana del siglo XX, Poitiers, CRLA-Archivos, 2005.

19. <http://www.mshs.univ-poitiers.fr/crla/contenidos/Archivos/accueil.html>.

20. Dans quelques cas, on a exploré des solutions ad hoc: dans l'édition de Martín Fierro, par exemple, on a confronté le texte établi avec la première version manuscrite conservé et on a enregistré les processus d'écriture dans des notes de bas de page.

21. <http://www.fahce.unlp.edu.ar/biblioteca/labiblioteca/archivo-digital-manuel-puig>.

22. On peut citer aussi d'autres recherches généticiennes produites au sein de l'UNLP : José Hernández, Vida del Chacho. Estudio Filológico Edición y Notas de María Celina Ortale, Buenos Aires, Ediciones del Dock, 2005 ; Julia Romero, «Ricardo Piglia, una poética de la reescritura », Orbis Tertius, $\mathrm{n}$ ำ16. 2010, <www.orbistertius.unlp.edu.ar>.

23. María Inés Palleiro, Fue una historia real. Itinerarios de un archivo, Buenos Aires, Instituto de Filología y Literaturas Hispánicas, Universidad de Buenos Aires, 2004.

24. Il s'agit d'une archive privée, qui se trouve dans l'établissement rural historique « Los Talas » (Luján, province de Buenos Aires), et le projet se réalise grâce au soutien de l'Université nationale de San Martín.

25. La maison d'édition institutionnelle (Unsam Edita) a publié déjà trois éditions critiques et génétiques préparées par Élida Lois : La guerra o el cesarismo en el Nuevo Mundo (2005); El crimen de la guerra (2007); Peregrinación de Luz del Día o Viaje y aventuras de la Verdad en el Nuevo Mundo (2008). 26. Voir, par exemple, «Una corresponsal de guerra en Buenos Aires. Acerca de las cartas inéditas de Ignacia Gómez de Cáneva a Juan B. Alberdi ", Nuevo Mundo Mundos Nuevos, Paris, Colloques, $2009<$ http://nuevomundo.revues.org/>.

27. <http://www.mshs.univ-poitiers.fr/crla/spip.php?article22>.

28. Pour un aperçu de ce travail, voir : Mariana Morón Usandivaras, «El taller de escritura de Alberdi. La génesis de un pensamiento », Recto/Verso, no 5, décembre 2009.

29. Voir, par exemple, «El fondo Daniel Moyano", Escritural. Écritures d'Amérique latine, nº 1, p. 406-449 (présentation de Marcelo Casarin).

30. Jean-Luc Nancy, The Birth to Presence, Palo Alto, Stanford University Press, 1993 ; Hans Ulrich Gumbrecht, Production of Presence: What Meaning Cannot Convey, Palo Alto, Stanford University Press, 2004.

31. Daniel Ferrer, «A crítica genética do século XXI será transdisciplinar, transartística e transemiótica ou não existirá », dans Fronteiras da Criação. VI Encontro Internacional de Pesquisadores do Manuscrito, dir. Mara Guasco, São Paulo, Annablume, 2000, p. 49-61. 


\section{RÉSUMÉS}

L'article brosse un tableau du paysage des études génétiques en Argentine. Au XIX ${ }^{\mathrm{e}}$ siècle, le juriste Juan Bautista Alberdi fut un précurseur de la critique génétique. Dans le premier tiers du $\mathrm{Xx}^{\mathrm{e}}$ siècle, ce pays dispose de quelques études de variantistica au sein d'une tradition philologique. En 1983, quasiment en même temps qu'arrivent d'Europe les premiers travaux génétiques, paraît, sous la plume d'Ana Maria Barrenechea, le premier ouvrage, consacré à une œuvre de Cortazar, qui témoigne d'un vrai renouvellement. Nourrie de traditions nationales, elle y développe une nouvelle conception de «texte» et introduit "l'avant-texte». Suit en 2001, d'Elida Lois, le premier manuel de critique génétique écrit en espagnol. Au plan des éditions, réalisés au sein de la collection "Archivos", paraissent une dizaine de volumes (dont certains sur CD-ROM). En même temps, l'Argentine a réalisé une réelle politique de sauvegarde de ses fonds littéraires; ainsi l'université de La Plata dispose du fonds immense de Manuel Puig, qui est maintenant mis en ligne.

This article gives an outline of Genetic studies in Argentina which has a philological tradition going back to the $19^{\text {th }}$ century, represented in particular by Jean Bautista Alberdi. In 1983, almost at the same time as the first European genetic studies reached Argentina, Ana Maria Barrenechea published a book on Cortazar's works that contributed a true renewal to this discipline. Imbued with national traditions, she developed a new idea of the text and introduced the "avant-texte". Then, in 2001, Elida Lois published the first genetic criticism textbook in Spanish. A dozen volumes of genetic editions have been published in the "Archivos" collection (some on CD-ROM). At the same time, Argentina has set up an effective protection policy for its literary collections. Thus La Plata University preserves Manuel Puig's huge collection and has put it online.

Este artículo presenta un panorama de los estudios genéticos en Argentina, donde ya en el siglo XIX las lecturas genéticas tuvieron un precursor en Juan Bautista Alberdi. Con los estudios filológicos de comienzos del siglo XX, aparecen estudios de variantística en medios académicos. En 1983, prácticamente al mismo tiempo que llegan de Europa los primeros estudios genéticos, se publica, con la firma de Ana María Barrenechea, el primer libro dedicado a un cuaderno de trabajo de Cortázar que representa una verdadera renovación: partiendo de las tradiciones nacionales, desarrolla una nueva concepción del "texto" e introduce el estudio del "pre-texto". Le seguirá, en 2001, el primer manual de crítica genética escrito en castellano, obra de Elida Lois. En lo que respecta a la edición, en el marco de la colección Archivos se publicaron una decena de títulos argentinos (algunos de los cuales acompañados por un CD-Rom). Paralelamente, la Argentina ha llevado a cabo una verdadera política de salvaguarda de sus fondos literarios : así, por ejemplo, la universidad de La Plata dispone de un enorme fondo Manuel Puig, que se encuentra actualmente disponible en línea.

Der Beitrag erlaubt einen Einblick in die Landschaft der Genetikstudien in Argentinien. Schon seit dem 19. Jahrhundert existiert in diesem Land eine philologische Tradition, mit Juan Bautista Alberdi als ihrem herausragenden Vertreter. Im Jahr 1983, sozusagen zeitgleich mit den ersten genetischen Arbeiten in Europa, erschien aus der Feder von Ana Maria Barrenechea das erste, einem Werk von Cortazar gewidmete Buch, das von wirklicher Erneuerung zeugt. Von nationalen Traditionen ausgehend, wird hier eine Neukonzeption von „Text“ entwickelt, und der Begriff des 
„avant-texte“ eingeführt. Dem folgt im Jahr 2001 Elida Lois' erstes Handbuch zur genetischen Kritik in spanischer Sprache. Was die Editionen betrifft, realisiert im Rahmen der Sammlung "Archivos“, erschienen mehr als zehn Bände (darunter einige auch auf CD-ROM). Zur selben Zeit entwickelte Argentinien eine wirkliche Sicherungspolitik für seine Literaturbestände ; dieser ist zu verdanken, dass die La Plata Universität große Bestände von Manuel Puig besitzt, die jetzt auch online verfügbar sind.

L'articolo schizza un quadro del paesaggio degli studi genetici in Argentina. Dal XIX secolo questo paese dispone di un modello di interpretazione filologica rappresentata principalmente da Juan Bautista Alberdi. Nel 1983, pressappoco nel periodo dell'arrivo dall'Europa dei primi lavori di genetica, appare, per opera di Ana Maria Barrenechea, il primo studio, dedicato a un testo di Cortazar, che testimonia un autentico rinnovamento. Nutrita di tradizioni nazionali, l'autrice vi sviluppa una nuova concezione del "testo" e introduce l'“avantesto". Segue, nel 2001, di Elida Lois, il primo manuale di critica genetica scritto in spagnolo. Nelle edizioni della collana "Archivos" appaiono una dozzina di volumi (alcuni su cd-rom). Allo stesso tempo l'Argentina ha realizzato una vera politica di salvaguardia degli archivi letterari; e l'università di La Plata possiede il fondo immenso di Manuel Puig, che viene ora messo in linea.

O artigo esboça um panorama dos estudos genéticos na Argentina. Desde o século XIX, o país estabeleceu uma tradição filológica, representada nomeadamente por Juan Bautista Alberdi. Em 1983, quase ao mesmo tempo que chegam da Europa os primeiros trabalhos genéticos, aparece, sob a pluma de Ana Maria Barrenechea, um primeiro estudo verdadeiramente renovador, consagrado a uma obra de Cortazar. Alimentando-se na tradição nacional, é aí desenvolvida uma nova concepção de "texto" e introduzido o conceito de "antetexto". Segue-se em 2001, de Elida Lois, o primeiro manual de crítica genética escrito em espanhol. No plano das edições, realizadas no quadro da colecção "Archivos", aparece uma dezena de volumes (alguns em CD-ROM). Ao mesmo tempo, a Argentina promove uma eficaz política de salvaguarda dos seus fundos literários; assim a universidade de La Plata dispõe do imenso espólio de Manuel Puig, agora disponível online.

\section{INDEX}

Mots-clés : critique génétique, Argentine, fonds manuscrits, état présent, édition génétique

\section{AUTEUR}

\section{ÉLIDA LOIS}

ÉLIDA LoIS est directeur de recherche au Conicet (Conseil national de recherches scientifiques et techniques, Argentine). Elle est aussi professeur à l'Université nationale de San Martín où elle dirige le Centre de recherches philologiques. Elle est l'auteur de Génesis de escritura y estudios culturales. Introducción a la crítica genética (2001), des éditions critiques et génétiques, annotées et préfacées de Don Segundo Sombra de R. Güiraldes (1988) et de Martín Fierro de J. Hernández (2001), pour la collection «Archivos », et des œuvres de J. B. Alberdi pour la Série « Alberdi » de Unsam Edita (2005-2010). Elle a écrit des nombreux articles sur la genèse textuelle d'œuvres d'auteurs argentins : Juana Manso, Leopoldo Lugones, Jorge Luis Borges.

elois[arobase]conicet.gov.ar 